\title{
The Extent of Chemically Enriched Gas around Star-forming Dwarf Galaxies
}

\author{
Sean D. Johnson ${ }^{1,2,6}$ (1) Hsiao-Wen Chen ${ }^{3}$ (1) John S. Mulchaey ${ }^{4}$, Joop Schaye ${ }^{5}$ (i), and Lorrie A. Straka ${ }^{5}$ (1) \\ ${ }^{1}$ Department of Astrophysical Sciences, 4 Ivy Lane, Princeton University, Princeton, NJ 08544, USA; sdj@astro.princeton.edu \\ ${ }^{2}$ The Observatories of the Carnegie Institution for Science, 813 Santa Barbara Street, Pasadena, CA 91101, USA \\ ${ }^{3}$ Department of Astronomy \& Astrophysics, The University of Chicago, 5640 S. Ellis Avenue, Chicago, IL 60637, USA \\ ${ }^{4}$ The Observatories of the Carnegie Institution for Science, 813 Santa Barbara Street, Pasadena, CA 91101, USA \\ 5 Leiden Observatory, Leiden University, P.O. Box 9513, NL-2300 RA Leiden, The Netherlands \\ Received 2017 September 15; revised 2017 October 7; accepted 2017 October 13; published 2017 November 15
}

\begin{abstract}
Supernova driven winds are often invoked to remove chemically enriched gas from dwarf galaxies to match their low observed metallicities. In such shallow potential wells, outflows may produce massive amounts of enriched halo gas (circumgalactic medium, CGM) and pollute the intergalactic medium (IGM). Here, we present a survey of the CGM and IGM around 18 star-forming field dwarfs with stellar masses of $\log M_{*} / M_{\odot} \approx 8-9$ at $z \approx 0.2$. Eight of these have CGM probed by quasar absorption spectra at projected distances, $d$, less than that of the host virial radius, $R_{\mathrm{h}}$. Ten are probed in the surrounding IGM at $d / R_{\mathrm{h}}=1-3$. The absorption measurements include neutral hydrogen, the dominant silicon ions for diffuse cool gas (T $\sim 10^{4} \mathrm{~K}$; Si II, Si III, and Si IV), moderately ionized carbon (C IV), and highly ionized oxygen (O VI). Metal absorption from the CGM of the dwarfs is less common and $\approx 4 \times$ weaker compared to massive star-forming galaxies, though $\mathrm{O}$ VI absorption is still common. None of the dwarfs probed at $d / R_{\mathrm{h}}=1-3$ have definitive metal-line detections. Combining the available silicon ions, we estimate that the cool CGM of the dwarfs accounts for only $2 \%-6 \%$ of the expected silicon budget from the yields of supernovae associated with past star formation. The highly ionized O VI accounts for $\approx 8 \%$ of the oxygen budget. As O VI traces an ion with expected equilibrium ion fractions of $\lesssim 0.2$, the highly ionized CGM may represent a significant metal reservoir even for dwarfs not expected to maintain gravitationally shock heated hot halos.
\end{abstract}

Key words: galaxies: dwarf - galaxies: halos - intergalactic medium - quasars: absorption lines

\section{Introduction}

To match the observed low metallicities (e.g., Lee et al. 2006) and stellar masses (e.g., McGaugh et al. 2010) of low- and intermediate-mass galaxies, models of galaxy evolution often invoke supernovae winds to prevent gas cooling and drive metal enriched gas out of star-forming regions (for a review, see Naab \& Ostriker 2017). In the shallow gravitational potential wells of dwarf galaxies, winds are expected to more easily reach large distances resulting in a decreased efficiency of wind recycling, a larger mass fraction of enriched halo gas (the circumgalactic medium, CGM), and pollution of the surrounding intergalactic medium (IGM) with metals.

Alternatively, interaction-related turbulent mixing and heating may remove enriched gas from galaxies without supernovae winds (e.g., Tassis et al. 2008). In this scenario, enriched material is not expected to escape from the host halo resulting in enriched CGM but pristine IGM around low-mass galaxies. A census of metal enriched CGM and IGM around dwarf galaxies therefore represents a key test of galaxy evolution models.

While the IGM and CGM are typically too diffuse to observe in emission with current facilities, UV absorption spectra from the Cosmic Origins Spectrograph (COS; Green et al. 2012) on the Hubble Space Telescope (HST) enable multi-phase surveys of the IGM/CGM at $z<0.4$ (for reviews see Chen 2016; Richter 2017; Tumlinson et al. 2017). For massive galaxies with stellar masses of $\log M_{*} / M_{\odot} \approx 10.5$, the CGM detected in the UV and X-ray at projected distances, $d$, less than the host

\footnotetext{
${ }^{6}$ Hubble \& Carnegie-Princeton Fellow.
}

halo virial radius, $R_{\mathrm{h}}$, constitutes a significant metal reservoir (e.g., Peeples et al. 2014; Miller \& Bregman 2015). For less massive galaxies of $\log M_{*} / M_{\odot}<9.5$, stars and the observed interstellar medium (ISM) only account for $\approx 20 \%$ of the expected metal yields from supernovae associated with past star formation (Tumlinson et al. 2017), but surveys of the CGM and IGM around dwarf galaxies are less extensive.

Recent studies of the CGM and IGM around intermediatemass $\left(\log M_{*} / M_{\odot} \approx 9.5\right)$ galaxies found little evidence for metal absorption at projected distances of $d / R_{\mathrm{h}} 0.7$ (Bordoloi et al. 2014; Liang \& Chen 2014). Moreover, the CGM of lowermass dwarf galaxies $\left(\log M_{*} / M_{\odot}<9.0\right)$ exhibit lower C IV (Burchett et al. 2016) and O VI (Prochaska et al. 2011) covering fractions than massive galaxies. Together, these observations suggest that galactic winds from dwarf galaxies may not pollute the CGM/IGM effectively or that their CGM is dominated by other ions. Additional observations of low-mass galaxies with measurents for a wider range of metal ions are needed for a more comprehensive view of the distribution and state of metal enriched gas around dwarf galaxies.

Here, we present a survey of diffuse absorbing gas around 18 star-forming field dwarf galaxies with a median stellar mass of $\log M_{*} / M_{\odot}=8.4$ at $z \approx 0.1-0.3$. Eight of these dwarf galaxies are probed by quasar spectra at projected distances of $d / R_{\mathrm{h}}<1$. Ten are probed at $d / R_{\mathrm{h}}=1-3$, enabling constraints on chemical enrichment beyond the nominal halo radius. The median stellar mass of our dwarf sample is over an order of magnitude lower than those of previous surveys with simultaneous measurements of absorption for the dominant silicon ions for diffuse cool gas at $\mathrm{T} \sim 10^{4} \mathrm{~K}$ ( $\mathrm{Si}$ II, Si III, and Si IV), moderately ionized carbon (C IV), and highly ionized 


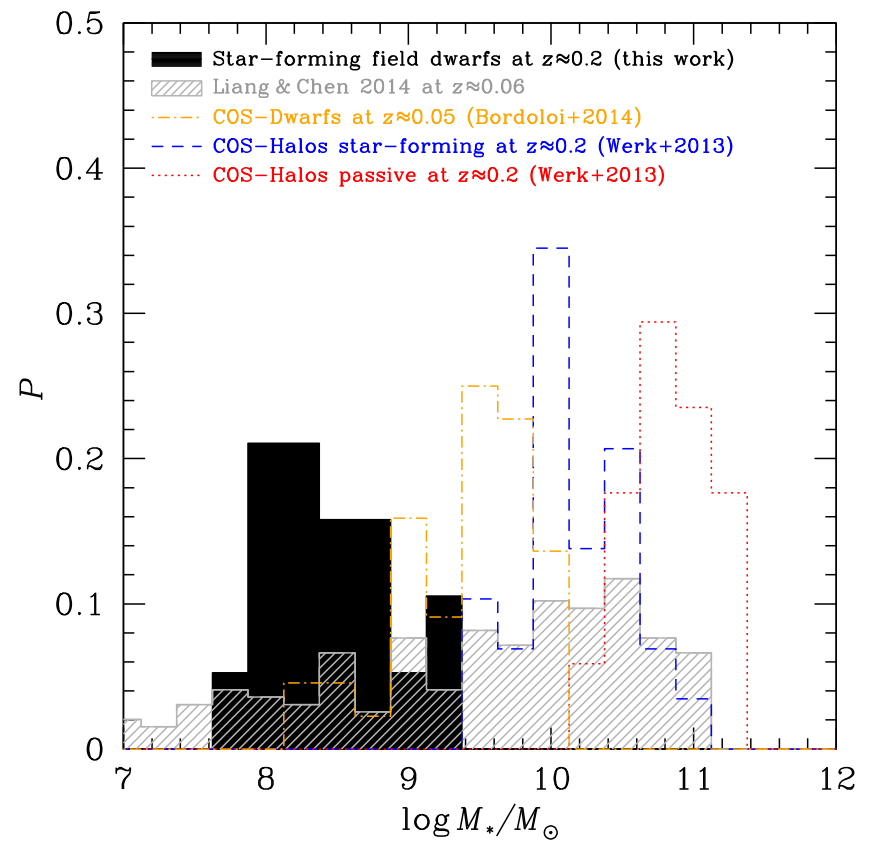

Figure 1. Stellar mass distribution of the star-forming field dwarf galaxies. For comparison, the star-forming and passive galaxies from the COS-Halos survey and intermediate-mass samples from COS-Dwarfs and Liang \& Chen (2014) are also shown.

oxygen (O VI) from COS spectra. The field dwarf galaxy sample is based on deep and highly complete redshift surveys ensuring that the dwarfs are not satellites of massive galaxies. Together, these data enable a comprehensive, controlled, and sensitive study of enriched CGM and IGM around dwarf galaxies.

This Letter proceeds as follows. In Section 2 we describe the dwarf galaxy sample and absorption spectroscopy. In Section 3 we characterize the CGM and IGM around the dwarfs. In Section 4 we discuss the implications of the survey.

Throughout the Letter we adopt a flat $\Lambda$ cosmology with $\Omega_{\mathrm{m}}=0.3, \Omega_{\Lambda}=0.7$, and $H_{0}=70 \mathrm{~km} \mathrm{~s}^{-1} \mathrm{Mpc}^{-1}$. We define a galaxy with luminosity of $L=L_{*}$ as having an $r$-band absolute magnitude of $M_{r}=-21.5$ based on the luminosity function from Loveday et al. (2012). All of the quoted magnitudes are in the $A B$ system.

\section{Sample and Data Description}

\subsection{Field Dwarf Galaxy Sample}

The dwarf galaxy sample is drawn from our absorption-blind spectroscopic surveys in the fields of quasars with high $S / N$ COS absorption spectra. The redshift surveys were conducted with the Magellan telescopes targeting galaxies of $r<23.5$ mag at angular distances of $\Delta \theta<10^{\prime}$ from the quasars. At redshifts of $z=0.1-0.3$, the magnitude limit corresponds to $L / L_{*}=$ $0.0025-0.025$. The details of the Magellan survey are published in Chen \& Mulchaey (2009) and Johnson et al. (2015). In summary the redshift errors are $\Delta v=30-60 \mathrm{~km} \mathrm{~s}^{-1}$, stellar masses assume a Chabrier (2003) initial mass function (IMF) and $g-r$ color dependent mass-to- $r$-band light ratio, halo masses assume the stellar-to-halo mass function from Kravtsov et al. (2014), and virial radii are defined as in Bryan \& Norman (1998).
To construct a field dwarf galaxy sample from our Magellan survey, we identified galaxies at $z=0.09-0.3$, of $L<0.1 L_{*}$, and at projected distances of $d / R_{\mathrm{h}}<3$ from the quasar sightline. The redshift cut ensures coverage of the desired absorption features and that the redshift surveys are sufficient to differentiate field dwarfs from satellites. The luminosity cut limits the sample to low-mass galaxies. The projected distance cut is chosen to be larger than $R_{\mathrm{h}}$ to examine enriched gas that may have escaped from the dwarfs. To ensure that the galaxy sample does not include satellites of massive galaxies, we removed galaxies that are within a projected distance of $500 \mathrm{kpc}$ and radial velocity of $\pm 300 \mathrm{~km} \mathrm{~s}^{-1}$ of a galaxy with $L>0.1 L_{*}$ (40\% sample reduction). We supplement our Magellan redshift survey with two field dwarf galaxies from Chen et al. (1998).

The properties of the resulting sample of 18 dwarf galaxies are summarized in Table 1. The sample spans a stellar mass range of $\log M_{*} / M_{\odot}=7.7-9.2$ with a median of $\log M_{*} / M_{\odot}=$ 8.4 (see Figure 1). All of the dwarfs galaxies have blue colors and multiple detected emission lines consistent with the low quiescent fraction of dwarfs in the field (e.g., Peng et al. 2010).

\subsection{COS Absorption Spectroscopy}

To study the CGM and IGM around the dwarf galaxies, we searched for H I and metal ion absorption in the COS quasar spectra within $\pm 300 \mathrm{~km} \mathrm{~s}^{-1}$ of the galaxy systemic redshifts. Increasing the search window to $\pm 600 \mathrm{~km} \mathrm{~s}^{-1}$ does not change the results for metal ions. Moreover, the velocity window is $>2 \times$ the estimated escape velocity in all cases assuming an Navarro-Frenk-White (NFW) profile (Navarro et al. 1996) with a concentration of 10 (Correa et al. 2015).

For galaxies with detected absorption, we measured the restframe equivalent widths, $W_{\mathrm{r}}$, of $\mathrm{H}$ I $\mathrm{Ly} \alpha, \mathrm{Si}$ II $\lambda 1260, \mathrm{Si}$ III $\lambda 1206$, Si IV $\lambda 1393, \mathrm{C}$ IV $\lambda 1548$, and O VI $\lambda 1031$ after local continuum normalization. For non-detections, we report $2 \sigma$ upper limits integrated over $90 \mathrm{~km} \mathrm{~s}^{-1}$ (the FWHM of O VI absorption from Johnson et al. 2015). When transitions are contaminated by systems at distinct redshifts, we placed limits based on other available transitions for the same ion accounting for oscillator strength and wavelength differences. The absorption equivalent widths and limits are in Table 1. For D7, we adopt absorption measurements from Prochaska et al. (2004).

\section{Results}

With the survey data described in Section 2, we characterize the CGM and IGM around star-forming field dwarf galaxies first by highlighting the two probed at the smallest projected distances in the sample in Figure 2. These two dwarfs, D1 and D2, are at $z=0.1229$ and 0.1613 , respectively, and have estimated stellar masses of $\log M_{*} / M_{\odot}=7.9$ and 8.1, respectively. Nondetection of [N II] $\lambda 6585$ lead to $2 \sigma$ upper limits on their ISM metallicities ofl $2+\log \mathrm{O} / \mathrm{H}<8.4$ (following Pettini \& Pagel 2004). D1 and D2 are probed by the quasar sightline at $d=16$ and $21 \mathrm{kpc}$ or $d / R_{\mathrm{h}}=0.2$ and 0.3 , respectively. For both D1 and D2, the COS spectra reveal strong $\mathrm{H}$ I absorption as well as Si III and O VI absorption. C IV absorption is detected for D1 but not covered by COS for D2. Si IV absorption is not detected for D2 but is not covered for D1. Neither dwarf is detected in Si II. Apart from a wing on the O VI line observed for D2, the metal absorption kinematics are consistent with bound motion in the host halos of D1 and D2, which have estimated escape velocities of $\approx 120 \mathrm{~km} \mathrm{~s}^{-1}$. 
Table 1

Summary of Dwarf Galaxy Properties and Associated Absorption

\begin{tabular}{|c|c|c|c|c|c|c|c|c|c|c|c|c|c|}
\hline \multirow[t]{2}{*}{ ID } & \multirow{2}{*}{$\begin{array}{l}\text { R.A. } \\
\text { (J2000) }\end{array}$} & \multirow{2}{*}{$\begin{array}{c}\text { Decl. } \\
\text { (J2000) }\end{array}$} & \multirow[b]{2}{*}{$z_{\text {gal }}$} & \multirow{2}{*}{$\begin{array}{r}M_{r} \\
(A B)\end{array}$} & \multirow[b]{2}{*}{$\log M_{*} / M_{\odot}$} & \multirow{2}{*}{$\begin{array}{c}d \\
(\mathrm{kpc})\end{array}$} & \multirow{2}{*}{$\begin{array}{c}R_{\mathrm{h}} \\
(\mathrm{kpc})\end{array}$} & \multicolumn{5}{|c|}{$W_{\mathrm{r}}$} & \multirow{2}{*}{$\underset{(\AA)}{\text { O VI } 1031}$} \\
\hline & & & & & & & & $\underset{(\AA)}{H}$ I $_{(\AA)}^{1215}$ & Si II $\underset{(\AA)}{(\AA)}$ & Si III $\underset{(\AA)}{1} 1206$ & $\underset{(\AA)}{\mathrm{Si} \mathrm{IV}}$ & $\mathrm{C}_{(\AA)}$ & \\
\hline D1 & $06: 35: 44.6$ & $-75: 16: 18$ & 0.1229 & -15.9 & 7.9 & 16 & 70 & $0.69 \pm 0.01$ & $<0.02$ & $0.13 \pm 0.01$ & $\ldots$ & $0.13 \pm 0.01$ & $0.12 \pm 0.01$ \\
\hline D2 & $06: 35: 45.7$ & $-75: 16: 23$ & 0.1613 & -16.2 & 8.1 & 21 & 80 & $0.77 \pm 0.10$ & $<0.01$ & $0.07 \pm 0.01$ & $<0.02$ & $\ldots$ & $0.13 \pm 0.01$ \\
\hline D3 & 02:35:07.7 & $-04: 02: 14$ & 0.2960 & -17.7 & 8.8 & 48 & 110 & $0.41 \pm 0.01$ & $<0.02$ & $<0.02$ & $<0.10$ & $<0.09$ & $<0.02$ \\
\hline D4 & 10:04:02.3 & $+28: 55: 12$ & 0.1380 & -17.2 & 8.2 & 56 & 80 & $0.69 \pm 0.01$ & $<0.01$ & $\ldots$ & $<0.03$ & $<0.04$ & $0.11 \pm 0.01$ \\
\hline D5 & $06: 35: 44.3$ & $-75: 15: 55$ & 0.1436 & -17.4 & 8.6 & 57 & 100 & $1.14 \pm 0.07$ & $<0.01$ & $\ldots$ & $<0.02$ & $\ldots$ & $<0.01$ \\
\hline D6 & $14: 37: 49.6$ & $-01: 47: 03$ & 0.1839 & -19.0 & 9.2 & 63 & 130 & $0.54 \pm 0.01$ & $<0.01$ & $<0.01$ & $<0.01$ & $<0.07$ & $0.13 \pm 0.01$ \\
\hline D7 & $04: 07: 49.3$ & $-12: 12: 16$ & 0.0923 & -16.1 & 8.3 & 72 & 90 & $0.47 \pm 0.01$ & $<0.01$ & $\ldots$ & $<0.02$ & $<0.01$ & $0.08 \pm 0.01$ \\
\hline D8 & $15: 47: 45.4$ & $+20: 51: 41$ & 0.0949 & -17.3 & 8.5 & 79 & 100 & $<0.01$ & $<0.02$ & $<0.01$ & $\ldots$ & $<0.03$ & $<0.02$ \\
\hline D9 & $15: 24: 23.3$ & $+09: 58: 58$ & 0.1391 & -14.9 & 7.7 & 84 & 60 & $0.06 \pm 0.01$ & $<0.02$ & $<0.01$ & $<0.04$ & $<0.02$ & $0.05 \pm 0.01$ \\
\hline D10 & $15: 55: 47.7$ & $+11: 11: 20$ & 0.1234 & -16.7 & 8.0 & 155 & 80 & $0.07 \pm 0.01$ & $<0.01$ & $<0.01$ & $<0.02$ & $<0.02$ & $<0.01$ \\
\hline D11 & $10: 05: 32.2$ & $+01: 33: 45$ & 0.1245 & -16.9 & 8.1 & 168 & 80 & $0.06 \pm 0.01$ & $<0.03$ & $<0.02$ & $<0.02$ & $<0.03$ & $<0.03$ \\
\hline D12 & $15: 24: 22.9$ & $+09: 59: 07$ & 0.2402 & -17.7 & 8.9 & 169 & 110 & $0.23 \pm 0.01$ & $<0.01$ & $\ldots$ & $<0.02$ & $<0.07$ & $<0.02$ \\
\hline D13 & $14: 37: 44.5$ & $-01: 48: 10$ & 0.1161 & -18.7 & 9.0 & 173 & 120 & $0.03 \pm 0.01$ & $<0.01$ & $<0.01$ & $<0.02$ & $<0.02$ & $<0.03$ \\
\hline D14 & $02: 35: 07.5$ & $-04: 03: 10$ & 0.1815 & -17.2 & 8.2 & 199 & 80 & $0.23 \pm 0.01$ & $<0.01$ & $<0.01$ & $<0.02$ & $<0.10$ & $<0.02$ \\
\hline D15 & $15: 24: 26.9$ & $+09: 57: 31$ & 0.1981 & -16.9 & 8.2 & 222 & 80 & $0.10 \pm 0.02$ & $<0.01$ & $\ldots$ & $<0.04$ & $<0.12$ & $<0.01$ \\
\hline D16 & $02: 28: 20.7$ & $-40: 56: 49$ & 0.1992 & -18.3 & 9.2 & 224 & 130 & $0.35 \pm 0.01$ & $<0.01$ & $<0.01$ & $<0.03$ & $\ldots$ & $<0.01$ \\
\hline D17 & $02: 28: 11.3$ & $-40: 56: 36$ & 0.2804 & -17.8 & 8.4 & 244 & 90 & $0.18 \pm 0.02$ & $<0.01$ & $<0.01$ & $<0.03$ & $\ldots$ & $<0.01$ \\
\hline D18 & $14: 37: 49.1$ & $-01: 48: 10$ & 0.2716 & -17.1 & 8.8 & 256 & 120 & $0.28 \pm 0.01$ & $<0.01$ & $<0.01$ & $<0.02$ & $<0.07$ & $<0.01$ \\
\hline
\end{tabular}




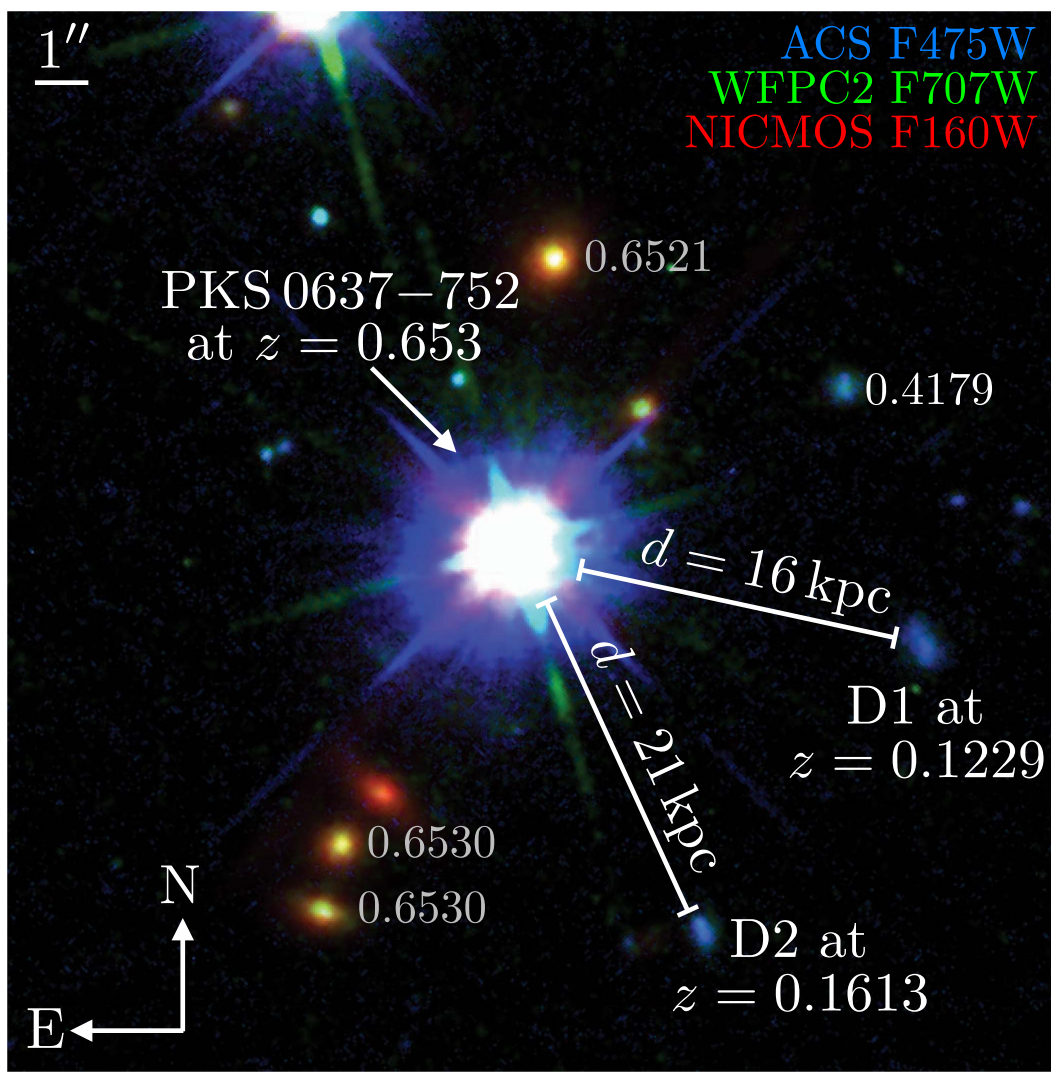

D1
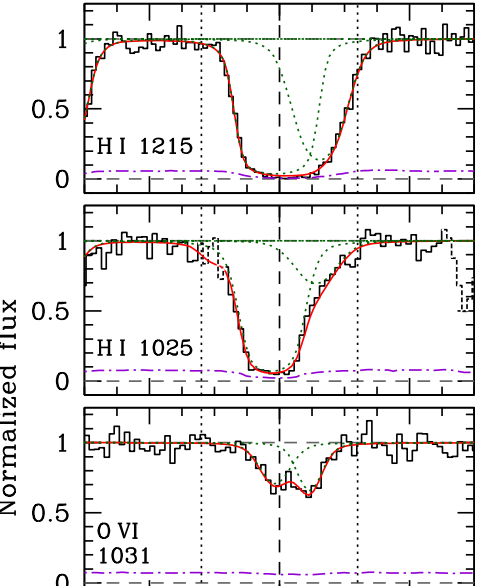

0

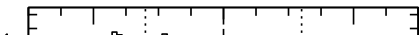

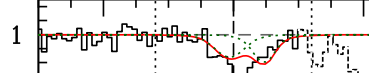

0.5 5 EO VI

0

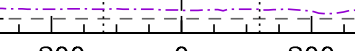

$\Delta v\left[\mathrm{~km} \mathrm{~s}^{-1}\right]$
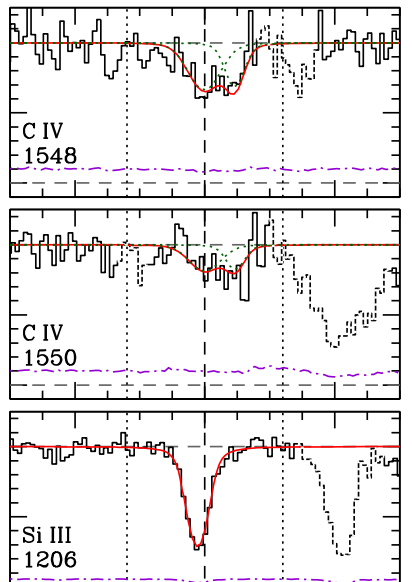

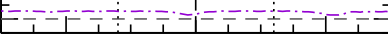

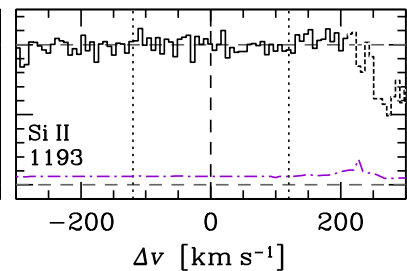

D2
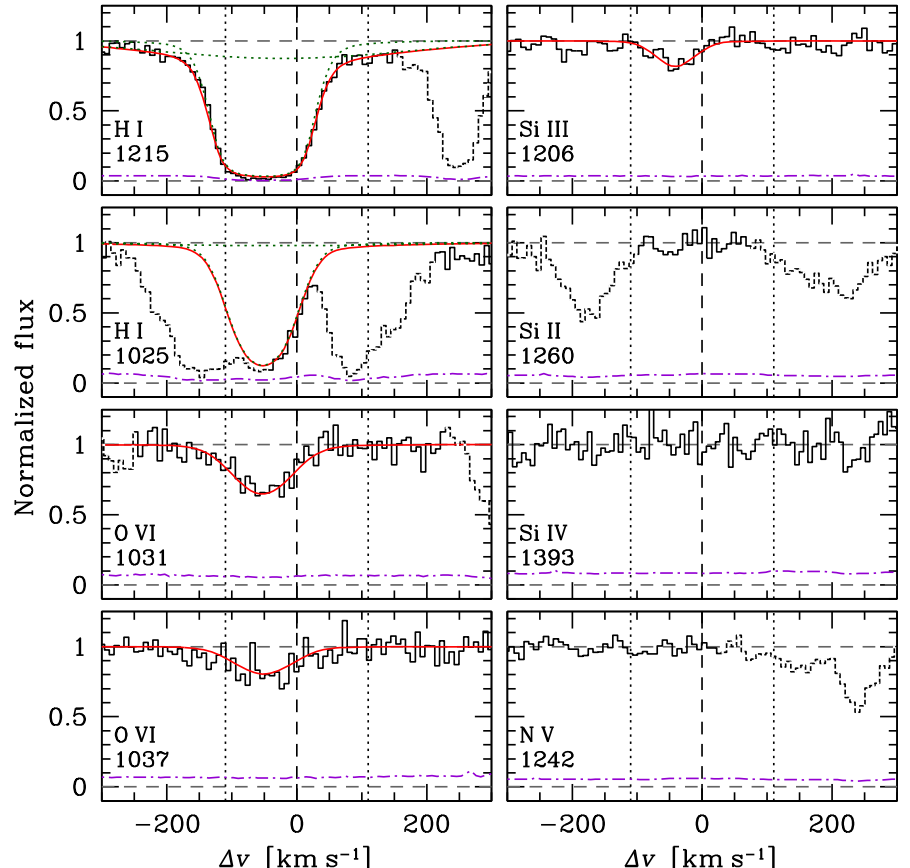

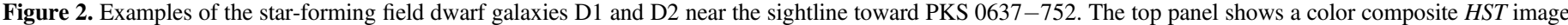

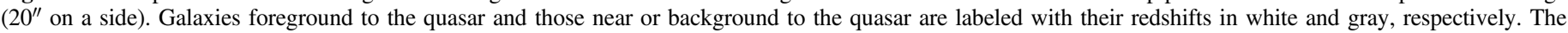

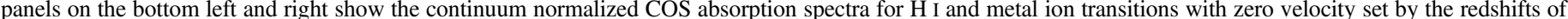

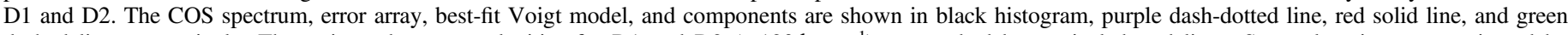

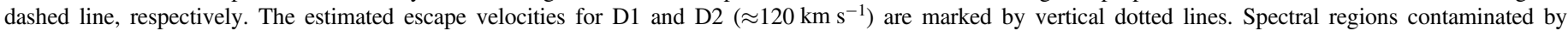
absorption from systems at distinct redshifts are dotted out.

To measure the column densities for the absorption associated with D1 and D2, we performed Voigt profile fitting with VPFIT (Carswell et al. 1987). For D1, the available Lyman series transitions are saturated so we estimate the column density range allowed by the data using the EMCEE Markov chain Monte Carlo package (Foreman-Mackey et al. 2013). The Voigt profile 
Table 2

Voigt Profile Fitting Measurements for D1 and D2

\begin{tabular}{|c|c|c|c|c|c|c|c|}
\hline Quasar Field & ID & Species & $\begin{array}{c}\Delta v \\
\left(\mathrm{~km} \mathrm{~s}^{-1}\right)\end{array}$ & $\begin{array}{c}b \\
\left(\mathrm{~km} \mathrm{~s}^{-1}\right)\end{array}$ & $\log N / \mathrm{cm}^{-2}$ & $\log N_{\text {tot }} / \mathrm{cm}^{-2}$ & Method \\
\hline \multirow[t]{8}{*}{ PKS 0637-752 } & D1 & $\mathrm{H} \mathrm{I}$ & $-12 \pm 3$ & $27_{-6}^{+2}$ & $15.7_{-0.2}^{+0.6}$ & $15.7_{-0.2}^{+0.6}$ & MCMC \\
\hline & $\cdots$ & $\mathrm{H} \mathrm{I}$ & $48 \pm 7$ & $41_{-3}^{+12}$ & $14.3_{-0.1}^{+0.3}$ & $\ldots$ & MCMC \\
\hline & $\ldots$ & Si II & $\ldots$ & $\ldots$ & $<12.15$ & $<12.15$ & $\mathrm{n} / \mathrm{a}$ \\
\hline & $\ldots$ & Si III & $-16 \pm 1$ & $15 \pm 1$ & $13.14 \pm 0.03$ & $13.14 \pm 0.03$ & VPFIT \\
\hline & $\ldots$ & C IV & $-7 \pm 12$ & $30 \pm 15$ & $13.53 \pm 0.19$ & $13.73 \pm 0.04$ & VPFIT \\
\hline & $\ldots$ & C IV & $39 \pm 8$ & $16 \pm 11$ & $13.31 \pm 0.30$ & $\ldots$ & VPFIT \\
\hline & $\ldots$ & O VI & $-12 \pm 12$ & $26 \pm 16$ & $13.79 \pm 0.19$ & $14.10 \pm 0.03$ & VPFIT \\
\hline & $\ldots$ & O VI & $41 \pm 8$ & $20 \pm 11$ & $13.80 \pm 0.18$ & $\ldots$ & VPFIT \\
\hline \multirow[t]{6}{*}{ PKS 0637-752 } & $\mathrm{D} 2$ & $\mathrm{H} \mathrm{I}$ & $-53 \pm 1$ & $46 \pm 1$ & $15.04 \pm 0.02$ & $15.06 \pm 0.02$ & VPFIT \\
\hline & $\ldots$ & $\mathrm{H} \mathrm{I}$ & $-23 \pm 30$ & $250 \pm 50$ & $13.66 \pm 0.05$ & $\ldots$ & VPFIT \\
\hline & $\ldots$ & Si II & $\ldots$ & $\ldots$ & $<11.8$ & $<11.8$ & $\mathrm{n} / \mathrm{a}$ \\
\hline & $\ldots$ & Si III & $-41 \pm 4$ & $35 \pm 6$ & $12.48 \pm 0.05$ & $12.48 \pm 0.05$ & VPFIT \\
\hline & $\cdots$ & Si IV & $\ldots$ & $\ldots$ & $<12.3$ & $<12.3$ & $\mathrm{n} / \mathrm{a}$ \\
\hline & $\ldots$ & $\mathrm{O} \mathrm{VI}$ & $-53 \pm 3$ & $62 \pm 5$ & $14.17 \pm 0.02$ & $14.17 \pm 0.02$ & VPFIT \\
\hline
\end{tabular}

measurements are reported in Table 2. For non-detections, we converted the equivalent width limits to column densities assuming the linear portion of the curve of growth.

The measured HI column densities for D1 and D2 of $\log N(\mathrm{H} \mathrm{I}) / \mathrm{cm}^{-2}=15.7_{-0.2}^{+0.6}$ and $15.06 \pm 0.02$ are lower than those of the Lyman limit systems $\left(\log N(\mathrm{H} \mathrm{I}) / \mathrm{cm}^{-2}>17.2\right)$ often observed for star-forming galaxies of $\log M_{*} / M_{\odot} \approx 10.5$ at similar $d / R_{\mathrm{h}}$ (e.g., Prochaska et al. 2017). The metal ion column densities observed for D1 and D2 are lower as well with $\log N(\mathrm{Si}$ III $) / \mathrm{cm}^{-2}=13.14,12.48$ and $\log N(\mathrm{O} \mathrm{VI}) / \mathrm{cm}^{-2}=$ $14.10,14.17$, respectively, compared to $\log N(\mathrm{Si}$ III $) / \mathrm{cm}^{-2} \gtrsim$ 13.5 (Werk et al. 2013) and $\log N\left(\mathrm{O}\right.$ VI) $/ \mathrm{cm}^{-2}=14.7$ (Tumlinson et al. 2011) for massive star-forming galaxies.

To investigate whether the modest absorption levels observed for D1 and D2 are typical of field dwarfs, we plot the measured rest-frame equivalent widths, $W_{\mathrm{r}}$, of H I Ly $\alpha$, Si II $\lambda 1260$, Si III $\lambda 1206$, Si IV $\lambda 1393$, C IV $\lambda 1548$, and O VI $\lambda 1031$ absorption as a function of $d / R_{\mathrm{h}}$ for the 18 dwarf galaxies in Figure 3. To increase the available sample for all but $\mathrm{O}$ VI, we also include star-forming field dwarfs of $\log M_{*} / M_{\odot}<9$ from the low-redshift surveys of Bordoloi et al. (2014) and Liang \& Chen (2014) after ensuring similar environmental criteria, stellar mass estimates, and absorption techniques. The stellar mass cut was chosen to a match the median stellar mass of our field dwarfs. Massive star-forming galaxies from Tumlinson et al. (2013), Werk et al. (2013), Liang \& Chen (2014), and Johnson et al. (2015) are shown for comparison.

Both $\mathrm{H}$ I and metal ion absorption around the dwarf galaxies is anti-correlated with $d / R_{\mathrm{h}}$. Despite the comparatively low $\mathrm{H} \mathrm{I}$ column densities observed for D1 and D2, the Ly $\alpha$ equivalent widths of the dwarf sample overlap with those of more massive galaxies (see the top left panel of Figure 3). This suggests that non-thermal motion contributes to the equivalent widths of Ly $\alpha$ observed around galaxies. Confirmation will require larger samples observed in the full Lyman series.

For metal ions on the other hand, the full sample confirms that the star-forming field dwarf galaxies exhibit modest absorption levels and lower covering fractions (the fraction of sightlines exhibiting detections to some equivalent width limit) compared to more massive galaxies. To better quantify this trend, we estimate the covering fractions for systems of
$W_{\mathrm{r}}>0.1 \AA$ for the silicon ions, C IV, and O VI both in the CGM at $d<R_{\mathrm{h}}$ and IGM at $d / R_{\mathrm{h}}=1-3$.

First we consider silicon ions. None of the dwarf galaxies are detected in Si II absorption with limits of $W_{\mathrm{r}}(\mathrm{Si}$ II 1260) $<$ 0.01-0.1 $\AA$ (top right panel of Figure 3 ) leading to $95 \%$ upper limits on the covering fraction of $\kappa_{\text {Si II }}<0.17(0.13)$ at $d / R_{\mathrm{h}} \leqslant 1\left(1<d / R_{\mathrm{h}} \leqslant 3\right)$. Only D1 exhibits Si III $\lambda 1206$ with $W_{\mathrm{r}}>0.1 \AA$ with similar limits (middle left panel of Figure 3) resulting in covering fraction estimates of $\kappa_{\mathrm{Si} \mathrm{III}}=0.10_{-0.03}^{+0.17}$ $(<0.14)$ at $d / R_{\mathrm{h}} \leqslant 1 \quad\left(1<d / R_{\mathrm{h}} \leqslant 3\right)$. Finally, none of the dwarf galaxies are detected in Si IV with similar limits (middle right panel of Figure 3 ) resulting in covering fraction limits of $\kappa_{\text {Si iv }}<0.19(0.13)$ at $d / R_{\mathrm{h}} \leqslant 1\left(1<d / R_{\mathrm{h}} \leqslant 3\right)$.

For moderately ionized carbon, only D1 and two lowredshift dwarf galaxies are detected in C IV with $W_{\mathrm{r}}>0.1 \AA$. All three of these are probed at $d / R_{\mathrm{h}}<0.6$. The COS spectra place limits of $W_{\mathrm{r}}(\mathrm{C}$ IV 1548$)<0.01-0.1 \AA$ for the other dwarfs (bottom left panel of Figure 3) leading to covering fraction estimates of $\kappa_{\mathrm{C} \text { IV }}=0.23_{-0.08}^{+0.15}(<0.14)$ at $d / R_{\mathrm{h}} \leqslant 1\left(1<d / R_{\mathrm{h}} \leqslant 3\right)$, consistent with low C IV covering fractions observed around dwarf galaxies by Burchett et al. (2016).

Considering highly ionized oxygen, D1 and D2, along two other dwarf galaxies, are detected in O VI with $W_{\mathrm{r}}>0.1 \mathrm{~A}$ and two show weaker OVI absorption (bottom right panel of Figure 3). Of the O VI detected dwarfs, only D9 is probed at $d / R_{\mathrm{h}}>1$, but the identification of the weak absorption as $\mathrm{O} \mathrm{VI}$ is tentative due to insufficient $\mathrm{S} / \mathrm{N}$ to detect the weaker $\lambda 1037$ doublet member. For the twelve dwarf galaxies without O VI detections, the COS spectra place upper limits of $W_{\mathrm{r}}(\mathrm{O}$ VI 1031$)<0.03 \AA$ or better. Based on this, we estimate an $\mathrm{OVI}$ covering fraction of $\kappa_{\mathrm{OVI}}=0.50_{-0.16}^{+0.16}(\leqslant 0.24)$ at $d / R_{\mathrm{h}} \leqslant 1\left(1<d / R_{\mathrm{h}} \leqslant 3\right)$ for systems of $W_{\mathrm{r}}>0.1 \AA$., consistent with non-detections of such systems among the three late-type field dwarfs from Prochaska et al. (2011) that are probed at $d / R_{\mathrm{h}}=0.5,1.3$, and 1.4.

\section{Discussion}

In a sample of star-forming field dwarf galaxies with a median stellar mass of $\log M_{*} / M_{\odot}=8.4$ probed at $d / R_{\mathrm{h}}<3$ by absorption spectra, we find that metal ion absorption 


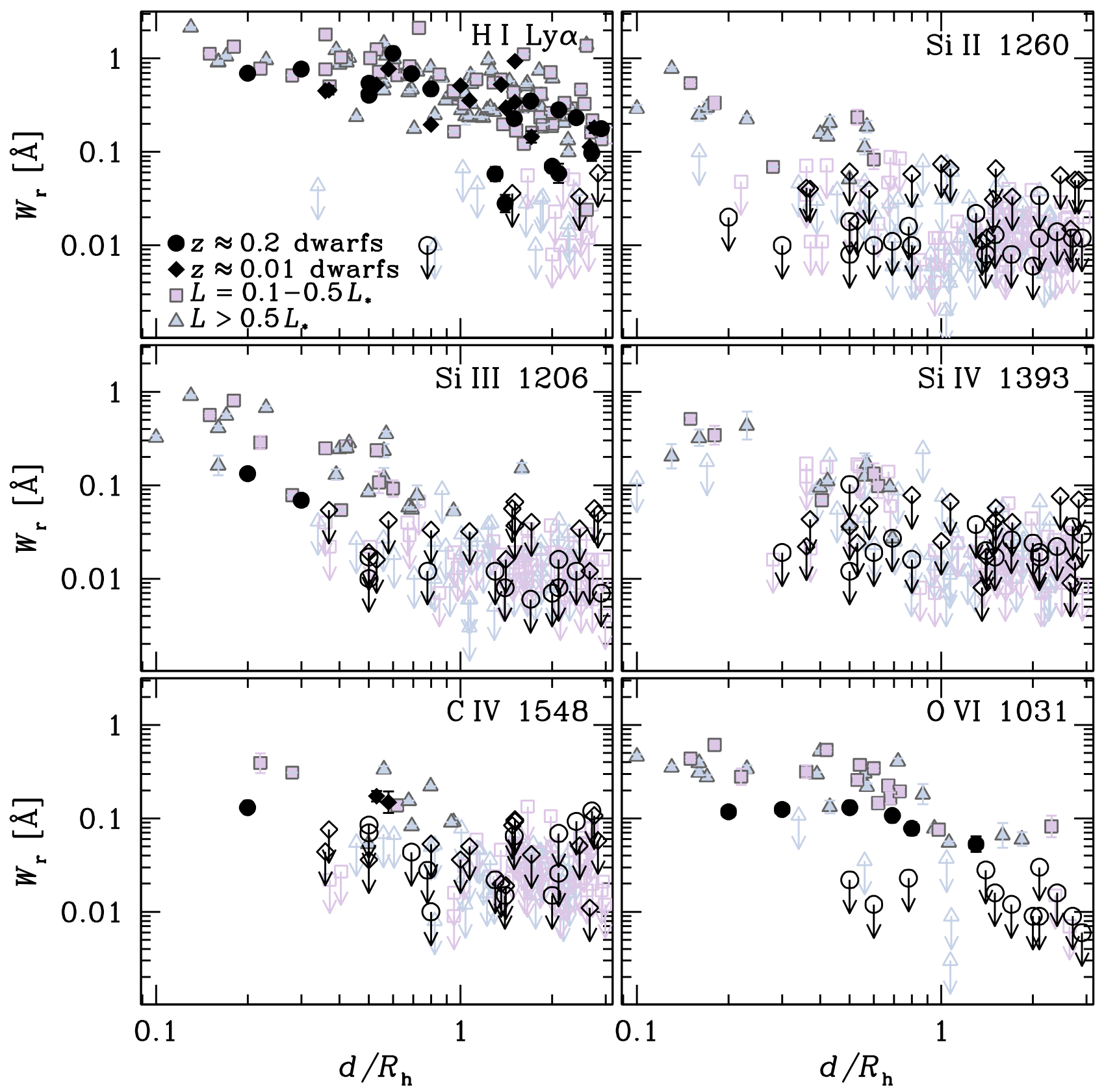

Figure 3. Rest-frame absorption equivalent width, $W_{\mathrm{r}}$, vs. virial radius normalized projected distance, $d / R_{\mathrm{h}}$, for $\mathrm{H}$ I Ly $\alpha$, Si II $\lambda 1260$, Si III $\lambda 1206$, Si IV $\lambda 1393$, $\mathrm{C}$ IV $\lambda 1548$, and $\mathrm{O}$ VI $\lambda 1031$. The star-forming field dwarf galaxies at $z \approx 0.2$ from this work are displayed as black circles and those of $\log M_{*} / M_{\odot}<9$ from the lowredshift surveys of Liang \& Chen (2014) and Bordoloi et al. (2014) are displayed as black diamonds. For comparison, star-forming galaxies of $0.1 L_{*}<L<0.5 L_{*}$ and $L>0.5 L_{*}$ from Werk et al. (2013) and Johnson et al. (2015) are shown in faded violet squares and faded blue triangles, respectively. Upper limits are $2 \sigma$ and shown as open symbols with downward arrows.

systems from a wide range of silicon ions ( $\mathrm{Si}$ II, Si III, and Si IV) and moderately ionized carbon (C IV) are less common and weaker than observed in the CGM of more massive starforming galaxies. Highly ionized oxygen absorption from O VI is common in the CGM of the dwarf galaxies but with lower covering fractions and absorption levels as well. For example, the two dwarf galaxies probed at $d / R_{\mathrm{h}}=0.2-0.3$ exhibit Si III, $\mathrm{C}$ IV, and $\mathrm{O}$ VI column densities that are $\approx 0.6$ dex lower than observed around more massive star-forming galaxies. None of the dwarf galaxies probed at $d / R_{\mathrm{h}}=1-3$ are definitively detected in metal ion absorption. These observations stand in contrast to analytic expectations and simulations (e.g., Shen et al. 2014; Christensen et al. 2016; Oppenheimer et al. 2016; Muratov et al. 2017; Wang et al. 2017) that predict more extended chemical enrichment and/or lower ionization states around dwarf galaxies.

CGM studies often scale projected distances by the estimated host virial radii to account for the self-similar nature of dark matter halo density profiles and approximate the observed mass-size scaling of the CGM (e.g., Chen et al. 2010). However, NFW profiles are not self-similar in projected density. An NFW profile with $c=10$ and $\log M_{\mathrm{h}} / M_{\odot}=$ 10.8 (corresponding to $\log M_{*} / M_{\odot}=8.4$ ) has projected densities that are $\approx 0.3$ dex lower than that of a halo of 
$\log M_{\mathrm{h}} / M_{\odot}=12.0$ at fixed $d / R_{\mathrm{h}}$. As this is smaller than the measured differences, mass-size scaling of the CGM alone cannot explain the decreased metal absorption around the dwarfs.

Stars and the ISM of dwarf galaxies account for $\approx 20 \%$ of the expected supernovae metal yields associated with past star formation (Tumlinson et al. 2017), and the CGM represents a potential reservoir for the remaining metals. We estimate metal mass of the CGM around dwarf galaxies in each ion, $M_{\text {ion }}$, as $M_{\text {ion }} \approx \pi R_{\mathrm{h}}^{2} m_{\text {ion }} \kappa_{\text {ion }}\left\langle N_{\text {ion }}\right\rangle$, where $m_{\text {ion }}$ is the atomic mass and $\left\langle N_{\text {ion }}\right\rangle$ is the mean column density among the detections. Adopting a virial radius of $R_{\mathrm{h}}=90 \mathrm{kpc}$ corresponding to a dwarf of $\log M_{*} / M_{\odot}=8.4$, mean column densities of D1 and D2, and observed covering fractions, we find ion masses of $M_{\mathrm{ion}} \approx 10^{4}, 3 \times 10^{4}$, and $3 \times 10^{5} M_{\odot}$ for Si III, C IV, and O VI, respectively. By assuming unity covering fractions and the limits for D2, we place limits of $M_{\text {ion }}<5 \times 10^{3}$ and $10^{4} M_{\odot}$ for Si II and Si IV. Together, Si II, Si III, and Si IV are the dominant silicon ions for diffuse gas at $T=10^{4}-10^{5} \mathrm{~K}$ (Oppenheimer \& Schaye 2013), so we estimate the total silicon mass in the cool CGM phase ofl-2.5 $\times 10^{4} M_{\odot}$.

To place these metal masses in context, we compare them to the metal budget from the yields of supernovae associated with the formation of $\log M_{*} / M_{\odot}=8.4$ worth of stars. Adopting an initial mass function normalized supernovae oxygen yield of $0.015 M_{\odot}$ per solar mass of star formation (see Peeples et al. 2014) and solar relative abundance (Asplund et al. 2009), we find that the cool CGM represents just $2 \%-6 \%$ of the silicon budget of the dwarf galaxies. The inferred ion masses from $\mathrm{C}$ IV and $\mathrm{O}$ VI absorption represent $\approx 2 \%$ and $\approx 8 \%$ of the expected carbon and oxygen budgets. $\mathrm{O}$ VI traces an ion with expected ion fractions of $\lesssim 0.2$ under photo- or collisional ionization equilibrium (Oppenheimer \& Schaye 2013) so this highly ionized CGM phase may account for $\approx 40 \%$ of the metal budget of the dwarfs, though we caution that higher ion fractions are possible (e.g., Vasiliev et al. 2015). Dwarf galaxies are not expected to maintain gravitationally shock heated hot halos (e.g., Correa et al. 2017), suggesting that the OVI arises from photoionized gas or other shock/ heating sources (e.g., Cen \& Chisari 2011).

In summary, our observations of modest metal ion absorption from Si II, Si III, Si IV, C IV, and O VI in the CGM and IGM around star-forming field dwarf galaxies suggest that (1) the CGM of field dwarfs is dominated by unexpectedly high ionization states, (2) galactic outflows are unexpectedly ineffective at enriching the cool-warm IGM surrounding lowmass galaxies at $z<0.3$, and/or (3) enriched outflows effectively mix with the IGM over time to reach lower metallicities than what are currently observable.

Dwarf galaxies are abundant and may contribute nonnegligibly to the low-redshift quasar absorption populations despite their modest CGM absorption. We estimate the number of metal absorption systems per unit redshift from field dwarf galaxies oflog $M_{*} / M_{\odot}=8-9$ as $\frac{d N}{d z}=\frac{c}{H_{0}} \Phi \sigma_{\text {ion }}$, where $\Phi$ is the number density and $\sigma_{\text {ion }}$ is the effective cross-section from the virial radius and observed covering fractions. Adopting $\Phi=0.017 \mathrm{Mpc}^{-3} \mathrm{dex}^{-1}$ based on the stellar mass function from Baldry et al. (2008) and discounting by the fraction dwarfs that are satellites (30\%; Zheng et al. 2007), we find $\frac{d N}{d z} \approx 0.1$ and $\approx 0.7$ for Si III and O VI systems with $W_{\mathrm{r}} \gtrsim 0.1 \AA$. Star-forming field dwarfs therefore account for $\approx 5 \%$ and $\approx 20 \%$ of such intervening $\mathrm{Si}$ III and O VI systems at low redshift (Thom \& Chen 2008; Tripp et al. 2008; Danforth et al. 2016).

We are grateful to Dave Bowen, Renyue Cen, Johnny Greco, Jenny Greene, and Fakhri Zahedy for comments on the draft. S.D.J. is supported by a NASA Hubble Fellowship (HST-HF251375.001-A). H.W.C. is partially supported by HST-GO14145.01.A. This research used data from the Magellan Telescopes and the NASA/ESA Hubble Space Telescope archive (NAS-5-2655: 6619, 10541,11508, 11520,11541,11692, 11741, 12025, 12038, 12264, 13024, and 13398), the NASA/IPAC Extragalactic Database, and the NASA Astrophysics Data System.

Facilities: HST, Magellan.

\section{ORCID iDs}

Sean D. Johnson (iD https://orcid.org/0000-0001-9487-8583 Hsiao-Wen Chen (i) https://orcid.org/0000-0001-8813-4182 Joop Schaye (iD https://orcid.org/0000-0002-0668-5560 Lorrie A. Straka (D) https://orcid.org/0000-0001-5892-6760

\section{References}

Asplund, M., Grevesse, N., Sauval, A. J., \& Scott, P. 2009, ARA\&A, 47 481

Baldry, I. K., Glazebrook, K., \& Driver, S. P. 2008, MNRAS, 388, 945 Bordoloi, R., Tumlinson, J., Werk, J. K., et al. 2014, ApJ, 796, 136

Bryan, G. L., \& Norman, M. L. 1998, ApJ, 495, 80

Burchett, J. N., Tripp, T. M., Bordoloi, R., et al. 2016, ApJ, 832, 124

Carswell, R. F., Webb, J. K., Baldwin, J. A., \& Atwood, B. 1987, ApJ, 319, 709

Cen, R., \& Chisari, N. E. 2011, ApJ, 731, 11

Chabrier, G. 2003, PASP, 115, 763

Chelouche, D., \& Bowen, D. V. 2010, ApJ, 722, 1821

Chen, H.-W. 2016, Outskirts of Galaxies, Astrophysics and Space Science Library, Vol. 434 (Berlin: Springer), 291

Chen, H.-W., Helsby, J. E., Gauthier, J.-R., et al. 2010, ApJ, 714, 1521

Chen, H.-W., Lanzetta, K. M., Webb, J. K., \& Barcons, X. 1998, ApJ, 498, 77

Chen, H.-W., \& Mulchaey, J. S. 2009, ApJ, 701, 1219

Christensen, C. R., Davé, R., Governato, F., et al. 2016, ApJ, 824, 57

Correa, C. A., Schaye, J., Wyithe, J. S. B., et al. 2017, MNRAS, 473, 538

Correa, C. A., Wyithe, J. S. B., Schaye, J., \& Duffy, A. R. 2015, MNRAS, 452, 1217

Danforth, C. W., Keeney, B. A., Tilton, E. M., et al. 2016, ApJ, 817, 111

Foreman-Mackey, D., Hogg, D. W., Lang, D., \& Goodman, J. 2013, PASP, 125,306

Green, J. C., Froning, C. S., Osterman, S., et al. 2012, ApJ, 744, 60

Johnson, S. D., Chen, H.-W., \& Mulchaey, J. S. 2015, MNRAS, 449, 3263

Kravtsov, A., Vikhlinin, A., \& Meshscheryakov, A. 2014, ApJ, submitted (arXiv:1401.7329)

Lee, H., Skillman, E. D., Cannon, J. M., et al. 2006, ApJ, 647, 970

Liang, C. J., \& Chen, H.-W. 2014, MNRAS, 445, 2061

Loveday, J., Norberg, P., Baldry, I. K., et al. 2012, MNRAS, 420, 1239

McGaugh, S. S., Schombert, J. M., de Blok, W. J. G., \& Zagursky, M. J. 2010, ApJL, 708, L14

Miller, M. J., \& Bregman, J. N. 2015, ApJ, 800, 14

Muratov, A. L., Kereš, D., Faucher-Giguère, C.-A., et al. 2017, MNRAS, 468, 4170

Naab, T., \& Ostriker, J. P. 2017, ARA\&A, 55, 59

Navarro, J. F., Frenk, C. S., \& White, S. D. M. 1996, ApJ, 462, 563

Oppenheimer, B. D., Crain, R. A., Schaye, J., et al. 2016, MNRAS, 460 2157

Oppenheimer, B. D., \& Schaye, J. 2013, MNRAS, 434, 1043

Peeples, M. S., Werk, J. K., Tumlinson, J., et al. 2014, ApJ, 786, 54

Peng, Y.-j., Lilly, S. J., Kovač, K., et al. 2010, ApJ, 721, 193

Pettini, M., \& Pagel, B. E. J. 2004, MNRAS, 348, L59

Prochaska, J. X., Chen, H.-W., Howk, J. C., et al. 2004, ApJ, 617, 718

Prochaska, J. X., Weiner, B., Chen, H.-W., et al. 2011, ApJ, 740, 91

Prochaska, J. X., Werk, J. K., Worseck, G., et al. 2017, ApJ, 837, 169 
Richter, P. 2017, Gas Accretion onto Galaxies, Astrophysics and Space Science Library, Vol. 43 (Berlin: Springer), 15

Shen, S., Madau, P., Conroy, C., Governato, F., \& Mayer, L. 2014, ApJ, 792, 99

Tassis, K., Kravtsov, A. V., \& Gnedin, N. Y. 2008, ApJ, 672, 888

Thom, C., \& Chen, H.-W. 2008, ApJS, 179, 37

Tripp, T. M., Sembach, K. R., Bowen, D. V., et al. 2008, ApJS, 177, 39

Tumlinson, J., Peeples, M. S., \& Werk, J. K. 2017, ARA\&A, 55, 389
Tumlinson, J., Thom, C., Werk, J. K., et al. 2011, Sci, 334, 948

Tumlinson, J., Thom, C., Werk, J. K., et al. 2013, ApJ, 777, 59

Vasiliev, E. O., Ryabova, M. V., \& Shchekinov, Y. A. 2015, MNRAS, 446, 3078

Wang, L., Dutton, A. A., Stinson, G. S., et al. 2017, MNRAS, 466, 4858

Werk, J. K., Prochaska, J. X., Thom, C., et al. 2013, ApJS, 204, 17

Zheng, Z., Coil, A. L., \& Zehavi, I. 2007, ApJ, 667, 760 